\title{
Cervantes y la novela moderna. Reflexiones desde la narratología y la teoría de la novela
}

\author{
Carlos García-Beidoya M. \\ Universidad Nacional Mayor de San Marcos
}

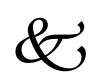

\section{Restmen}

Don Quijote de la Mancha de Miguel de Cervantes es comunmente considerada como la primera novela modema en la historia de la literatura. Diferentes opiniones sobre este punto son discutidas en este artículo. Usando categorías de la narratología y la teoría de la novela (Lukcas y Bajtin en particular) nuevos argumentos son propuestos para demostrar la modernidad de la obra maestra de Cervantes.

Palabras claves: Miguel de Cervantes, Don Quijote de la Mancha, novela modema, narratología.

\begin{abstract}
Miguel de Cervantes's Don Quixote is widely considered the first modem novel in the history of Literature. Different opinions on that topic are discussed here. Using categories from narratology and theory of the novel (Iukcas and Bajtin in particular), new arguments are provided in favor of the modemity of Cervantes's masterwork.

Key words: Miguel de Cervantes, Don Quijote de la Mancha, modem novel, namatology.
\end{abstract}

1. En su conocido libro Las palabras y las cosas, Michel Foucault proclama terminantemente a Don Quijote como la primera dora literaria modema. Aunque sus argumentos puedan resultar discutibles y hasta especiosos, su opinión sobre la modemidad de la novela cervantina es ampliamente compartida. Por su parte, Ian Watt destaca al personaje de don Quijote como uno de los mitos fundacionales del individualismo moderno, al lado de Fausto, Don Juan y Robinson Crusoe. Partiendo de algunos 
planteamientos de la narratología y la teoría de la novela, ( Luckas y Bajtin) en este artículo me propongo situar someramente a Cervantes y en particular a su novela Don Quijote, en el proceso de conformación de la novela modema. Una de las aspiraciones mayores de la novela modema es la ambición de unidad, la búsqueda de una forma narrativa o composición cerrada, en la cual haya una relación de necesidad entre las partes y el todo, de tal manera que cada episodio adquiera su sentido en relación al todo novelesco, y que a éste no se le puedan ni añadir ni quitar partes sin atentar contra su equilibrio organizativo. Este fue el modelo ideal de la novela decimonónica, que alcanzó tal vez su expresión más acabada, por lo menos en este aspecto de la unidad de composición, en Madame Bovary, de Flaubert. ¿Qué papel cumplió la obra cervantina en el desarrollo de este modelo?

Ia Edad Media tardía desarrolló dos modelos narrativos principales: la novela de caballería y la novel la boccacciana. La primera forma era un ejemplo característico de composición abierta o aditiva: se organizaba mediante la sucesión de aventuras y peripecias, susceptibles de alargarse indefinidamente mediante la inserción de episodios y personajes secundarios; el temue hilo de unidad lo proporcionaba el recorrido biográfico del protagonista principal. Tal vez el ejemplo más acabado de este modelo fue el Lancelot en prosa, larguísima novela caballeresca (casi tan larga como A la recherche du temps perculu de Proust) que fue quizá la más popular de la baja edad media europea. En España, y en un periodo más tardío, el ejemplo clásico es el respetablemente largo Amadís de Gaula, con las complejas aventuras del héroe epónimo, de su hermano Galaor, y luego de su hijo Esplandián en toda una serie de continuaciones.

La otra forma narrativa era la novella, llevada a su perfección por Boccaccio en base a los modelos del cuento popular, el cuento oriental y el apólogo greco-latino. En tanto forma narrativa breve, la novella presentaba la ventaja de una fuerte unidad, pero estaba limitada en su capacidad de abarcar universos narrativos más complejos. Mediante la incorporación de un conjunto de novellas en un marco narrativo más vasto, Boccaccio intentó en su Decamerone superar esa limitación.

El Renacimiento heredó estos dos modelos narrativos y en general trabajó en base a ellos sin introducir demasiadas modificaciones. La 
novella fue un género predilecto de la época, desde el Heptameron de Marguerite de Navarre, hasta las Nbvelas ejemplares del propio cervantes. Ias formas narrativas largas prefirieron la composición de tipo aditivo. tal como es visible en doras tan características como el Orlando furioso de Ariosto o en Gargantua et Pantagnuel de Rabelais. La única forma narrativa nueva creada por el Renacimiento italiano fue la novela pastoril, en la que se fundía la tradición clásica de la poesía eglógica con una variante especialmente ideal izante y artificial del amor cortés. Sin embargo, se desarrollaron algunos experimentos iniciales de estructura cerrada, y tales desarrollos tuvieron lugar justamente en España. La Celestina puede verse en términos generales como una obra de composición más bien cerrada, aunque ciertos episodios resulten algo marginales a la estructura central, organizada en torno a dos ejes: la relación amorosa Calisto/Melibea, y el conflicto amos/criados. La Celestina, lamentablemente, no tuvo en este aspecto como en otros muchos, un impacto inmediato sobre la narrativa posterior. ${ }^{2}$ Más repercusiones tuvo la novela picaresca, cuyo paradigma, el Lazarillo de Tormes, parece a primera vista una sucesión aditiva de peripecias, pero que, tal como lo ha destacado la crítica (Claudio Guillén, Francisco Rico, Lázaro Carreter, entre otros), adquiere una unidad superior por el punto de vista desde el aal se relatan los acontecimientos, de tal modo que la vida del pícaro cobra su sentido cabal en función de su situación final.

Tal era la variedad de formas narrativas de las que disponía cervantes para desarrollar su creatividad, y es posible afirmar que las exploró todas. Su carrera como narrador comienza en 1585 con la publicación de La Galatea, expresión de la narrativa pastoril que gozaba entonces de toob su prestigio. La composición de la obra es bastante laxa, con un hilo narrativo central en tomo a los protagonistas Elicio y Galatea, interrumpido constantemente por las peripecias de los demás pastores, pareciendo casi que el autor quiso trazar un cuadro de las diversas posibilidades de relación amorosa. Es interesante constatar que la dora permaneció inconclusa y que Cervantes siempre acarició (incluso en su patética dedicatoria del Persiles y Segismumda) la esperanza de concluirla en una segunda parte. En las Novelas ejemplares, Cervantes se propone explícitamente aclimatar ese género italiano a la tradición española. Sin embargo, prescinde del marco narrativo externo como 
modo de agrupar al conjunto de historias, conciente de la artificialidad de ese procedimiento. En cambio, tal como lo señala Emilio Orozco Díaz, intenta darles una unidad de intención, de carácter moral ${ }^{3}$.

Pero es evidentemente en el Quijote donde podemos ver los aportes més interesantes de Cervantes. El contraste entre la primera y la segunda parte es en este sentido esclarecedor. La primera parte se mueve todavía dentro del tipo de composición aditiva o abierta. Gran parte de la crítica admite actualmente además que la primera salida debió constituir originalmente una novella autónoma, que probablemente hubiera encontrado luego su lugar entre las Novelas ejemplares (allí don Quijote le habría hecho muy buena compañía al Licenciado Vidriera) . En torno al eje narrativo conformado por la pareja protagónica de Sancho y don Quijote, la primera parte de la novela se organiza en una sucesión de episodios, bastantes de los cuales tienen una relación más bien tenue con la trama principal. La segunda parte en cambio tiene una composición algo más cerrada, tanto por la presencia de un desenlace final, la recuperación del juicio y la muerte de don Quijote, que iluminan a nueva luz el conjunto de la dbra, como por el hecho de que en esta parte los episodios intercalados tienen todos una relación directa y orgánica con la pareja protagónica4 . La segunda parte del Quijote representa pues a este nivel un paso importante en el desarrollo de la novela moderna, paso del cual el propio Cervantes parece no haber tenido plena conciencia, pues en el Persiles y Segismunda retoma a una forma básicamente aditiva, con la sucesión de aventuras de los protagonistas y los mumerosos personajes secundarios, aunque en el caso de su última novela, a diferencia de La Galatea, hay un final que permite cerrar narrativamente la novela.

Durante el siglo XVI y la primera mitad del XVII, los desarrollos más interesantes de la narrativa de Occidente se dieron en España. Sin embargo, la reacción señorial que paralizó las fuerzas socioeconómicas activas en el XVI ejerció un efecto homólogo en el campo cultural ${ }^{5}$. Vale la pena notar que en España más que en ninguna otra parte de Eurropa se desarrolló una versión incipiente de la industria cultural de masas, con la vasta popularidad de formas narrativas como las novelas de caballería y la picaresca, y la aún más amplia de la comedia, y que también esta faceta sufre un colapso hacia mediados del XVII, 
paralizándose la cultura española en un estéril ritualismo cortesano. En consecuencia, los desarrollos posteriores en el campo de la novela no pasarán por España, sino por Francia e Inglaterra. En Francia se irá desarrollando en el XVIII el modelo de la novela sicológica sentimental con Manon Lescaut de Prévost y sobre todo con el carácterístico género de la novela epistolar, como en La nouvelle Héloïse de Rousseau o Les liasons dangereuses de Laclos, que luego aprovechará Goethe en su Werther. Ia industria del libro al canzará su mayor desarrollo en Inglaterra, que dará con la Pamela de Richardson (también de tipo epistolar) el primer best-seller modemo. En el siglo XIX, en Francia principalmente, pero también en Inglaterra y Rusia, la novela de composición cerrada se constituirá en la forma narrativa característica de la burguesía triunfante.

2. En su discusión del Quijote, Félix Martínez Bonati ${ }^{6}$ insiste mucho en que esta obra no puede considerarse en sentido estricto una novela moderna. Apunta que «Uno de los atributos esenciales de la novela modema, a pesar de todas sus variedades, es su homogeneidad interna. Para cada una hay una ley, y solamente una ley, que determina su universo imaginario». ${ }^{7}$ Novela moderna es para él sinónimo de novela realista decimonónica: lo que se pone en cuestión entonces es el realismo del Quijote. Para Martínez Bonati, la presencia constante del elemento cómico relativiza el realismo, presente en la obra sobre todo en los aspectos vinculados a la vida cotidiana. El Quijote es ante todo una obra metaficcional, en la cual todos los modelos literarios quedan cuestionados, en la que los procedimientos narrativos son puestos en evidencia, de manera que ni los códigos de la novela caballeresca, ni los de la pastoril, la picaresca o la novella resultan validados. Novela autoirónica, al poner en tela de juicio los géneros aceptados en su época, en opinión de Martínez Bonati despeja el camino a la novela modema, pero no la crea propiamente, puesto que no es una obra organizada en tomo a los rígidos principios de verosimilitud del real ismo del XIX.

En cambio, my distinta es la qpinión de Stephen Gilman, ${ }^{8}$ quien dedicó toob un libro a demostrar que el aujjote es la dbra iniciadbra de la novela, en el sentido modemo del término. Gilman sustenta su planteamiento en la distinción ya clásica en la crítica anglosajona entre ramance y novel. 
Northrop Frye la resume del siguiente modo: «La diferencia esencial entre novela y ronance reside en la creación de la caracterización. Ell romancista no intenta crear «gente de verdad» sino figuras estil izadas que se expanden como arquetipos psicológicas». ${ }^{9} \mathrm{Y}$ agrega luego, intentando vincular cada forma con un universo social: «Las afinidades sociales del romance, con su grave idealización del heroísmo y la pureza, se dan con la aristocracia... No es sorprendente, por tanto, que un importante tema en la novela, más burguesa, sea la parodia del romance y sus idealess ${ }^{10}$. Partiendo de esta perspectiva (y empleando la misma dicotonía terminológica) , la conclusión final de Gilman es la siguiente: «Al igual que colón, sin saber con exactitud dónde, Cervantes había llegado a un muevo continente que después se denominaría novelas ${ }^{11}$.

Sin dejar de reconocer la pertinencia de muchos de los planteamientos de Martínez Bonati, me inclino más a compartir el punto de vista de Stephen Gilman. Con la ayuda de algunos de los teóricos mayores de la novela, podremos deslindar mejor de qué manera el Quijote abre el camino a la novela modema. En su clásico Teoría de la novela, Georg Lukács define el género novelístico, en oposición a la epopeya, como la historia de un héroe problemático en un mundo problemático. Mundo problemático, pues las inamovibles barreras sociales de las sociedades tradicionales entran en ebullición, porque sus valores ya no son incuestionables y porque los individuos ya no tienen asignado un lugar en el cosmos social, sino que tienen que apoderarse de alguno en el caos humano.

La forma interna de la novela ha sido entendida como el proceso del individuo problemático en búsqueda de sí mismo, el camino desde un pesado cautiverio en una realidad meramente presente -una realidad que es heterogénea en sí misma y carente de significación para el individuo- hacia un claro auto-reconocimiento. ${ }^{12}$

El protagonista de la novela es pues un héroe problemático en busca de su lugar en el mundo. Esta condición implica que el héroe sufre transformaciones decisivas a lo largo de la diégesis: transformaciones en su condición social, transformaciones en su escala de valores (en sus convicciones) y transformaciones en su propia identidad síquica. Lukács postula dos modalidades de inadecuación del héroe al mundo exterior: el idealismo abstracto y el romanticismo de la desilusión. En el primer 
caso, el alma del héroe, dominada por un ideal excesivamente dosesivo, resulta demasiado estrecha para enfrentar un mundo complejo. Fll héroe sin embargo no se halla internamente desgarrado, sino que, poseído por su ideal, se enfrenta a un mundo exterior degradado. El paradigma de este tipo de héroe problemático es para lukács don Quijote, cuyas dosesiones cabal lerescas se estrellan contra un mundo exterior prosaico. Ia segunda modalidad es el romanticismo de la desilusión, en la que el alma del héroe resulta más amplia y rica que el mundo exterior degradado. Como ejemplo supremo de esta modalidad propone Lukács a la Education sentimentale de Flaubert. Resulta indudable que en el Quijote asistimos a un conflicto de valores que enfrenta a héroe y mundo, e incluso pienso que los personajes principales, don quijote y Sancho, sufren una transformación mayor de la que cree Lukács. En todo caso, ya no nos hallamos ante personajes meramente planos como los de la novela de caballería, la pastoril, o incluso la novela bizantina (el Persi les del propio Cervantes), sino ante personajes cuya dinámica interior resulta mucho más compleja, aún si está mediatizada por la ironía y la comicidad.

El otro teórico cuyos aportes pueden ayudarnos a examinar la contribución de Cervantes al desarrollo de la novela moderna es indudablemente Bajtin. ${ }^{13}$ Bajt in nos habla de la existencia de dos líneas en el desarrollo de la novela europea. En una primera, a la que se pootría denominar homofónica, las potencialidades dialógicas del género están atrofiadas: percibimos fundamentalmente una voz (la del narrador) y un universo axiológico, aunque nunca es posible hablar en términas estrictos de monologi.smo, pues toob discurso, aun el más homogéneo, lleva en su interior los ecos de otros disarsos (de la intentextualidad) . Fin la segunda, las posibilidades dialógicas del género se magnifican hasta alcanzar la vasta riqueza de la polifonía, en la que multiplicidad de voces y valores encontrados dialogan en el texto, relativizándose mutuamente. La primera línea, inicialmente dominante, estaría representada por la novela de aventuras de la Grecia antigua, la novela caballeresca, la novela barroca de la prueba del hérce, la novela sentimental del XVIII (Richardson, Rausseau) o el folletín decimonónico. Ia segunda tendría su antecedente primitivo más claro en un género como la Sátira Menipea, y daría sus primeros fnutos solitarios en el Satiricán de Petronio y en el Asno de oro de Apuleyo; se nutriría de ciertos elementos de la tradición narrativa popular medieval; alcanzaría un primer 
esplendor con Rabelais, la picaresca española, el Quijote y la novela cámica inglesa (Fielding, Steme) , para finalmente constituirse en la manifestación novelesca dominante con el realismo decimonónico, ayya más alta expresión es, para Bajtin, Dostoyevski.

Fhel Quijote, la manifestaciónmás evidente del dialogismo son las continas conversaciones entre don Quijote y Sandho, en las cuales ninguna de las dos voces recibe una sanción autorial que le dé un peso mayor, sino que ambas merecen ser evaluadas críticamente por el lector: la voz de la verdad parece hablar a veces por intermedio de la sabiduría popular de Sancho, a veces por intermedio de los conocimientos librescos de don Quijote, aunque más frecuentemente ambas voces se ven relativizadas por una distanciación cómica. Las jerarquías sociales tradicionales quedan así cuestionadas, pero no simplemente invertidas a la manera de la liberación fugaz del camaval, que implica un pronto retomo al orden jerárquico: ambas voces narrativas ocupan lugares fluctuantes en relación con la organización senéntica de la dora.

Otro mecanismo que incrementa la polifonía textual es el juego de los múltiples narradores/»autores», que da lugar a lo que muchos críticos han denominado perspectivismo. ${ }^{14} \mathrm{El}$ supuesto «auton», Cide Hamete Benengeli, los diversos narradores de las historias intercaladas, introducen una multiplicidad de puntos de vista en el desarrollo discursivo del texto, frecuentemente remitiéndose irónicamente los unos a los otros. Son clásicas los juegos autoirónicos, como las referencias a la pérdida de los papeles de Cide Hamete, o al posible carácter apócrifo de ciertos capítulos. Finalmente señalo un tercer mecanismo polifónico, la constante reflexión metaficcional, la puesta en evidencia del carácter literario del texto, desde los simples procedimientos de la discusión de tópicos literarios en distintos episodios (la quema de libros, las discusiones con el caballero del Verde Gabán y su hijo, etc. ) , hasta formas mucho más complejas, sobre todo en la segunda parte, en la que los dos protagonistas son tratados por todas las personas que encuentran en su peregrinar como personajes de novela, conocidos a través de la lectura de la primera parte.

Es sin duda a este nivel, al transgredir las barreras tradicionales de los decoros genéricos, al construir una forma narrativa que aprovecha todos los géneros narrativos propios de la época, pero que escapa completamente a sus marcos, construyendo un texto totalmente acanónico, que el Quijote cumple una labor cuestionadora que abre el 
camino a la novela moderna. Pero es también evidente que su autoironía metaficcional resultará más afín a la novela del siglo XX, empeñada en escapar a los estrechos códigos de verosimilitud y a las formas estructuralmente cerradas predominantes en el realismo decimonónico.

\section{Notas}

1 «La composición designa genéricamente cierta organicidad de la dbra artística, es decir, el principio de que la dora artística no es un conjunto arbitrario y caótico de elementos inconexos, sino un todo con cohesión, dotado de una economía intema que impone conexiones de interdependencia entre esos elementos» (Reis y Lopes. Diccionario de nacratología, 45).

2 Sobre La Celestina y su impacto sobre el desarrollo de la novela modema, puede consultarse mi artículo (publicado en ocasión de cumplirse los quinientos años de la primera edición de esa dora clásica) «La celestina en la encrucijada».

3 Cervantes y la novela del barroco, 50-51.

4 Sobre estos aspectos, resulta ilustrativo el análisis de Segre en «Costruzioni rettil inee e costnuzioni a spirali nel Don chisciotte. Véase también el libro de Riley Introducción al Quijote, capítulos 7, 8 y 9.

5 Sobre este fenómeno, véase el libro de Maravall La aultura del Barroco.

6 Vease su libro Don Quixote and the Poetics of the Novel. Este importante libro, a pesar de haber sidb escrito en castellano, se publicó primero en inglés.

7 Don Quixote and the Poetics of the Novel, 64. (Mi traducción) .

8 La novela según Cervantes. A diferencia de la dbra de Martínez Bonati, este libro se escribió originalmente en inglés y se publicó primero en esa lengua en 1989.

9 Anatomy of Criticism, 304 (mi traducción).

10 Anatany of Criticism, 306 (mi traducción).

11 La novela según Cervantes, 182.

12 The Theory of the Novel, 80 (mi traducción) .

13 Consúltese los trabajos incluidas en su libro Esthétique et théorie du raman.

14 El término fue acuñado por Spitzer en su conocido estudio «Perspectivismo lingüístico enel Quijotes.

\section{Referencias bibliográficas}

AUERBACH, Erich(1982) : Mimesis. Fondo de Cultura Económica, México. BAKHIINE, Mikhäll (1987) : Esthétique et théorie du roman. Gallimard, Paris.

FOUCAULT, Michel (1968) : Las palabras y las casas. Siglo XXI, Madrid. 
FRYE, Northrop (1957) : Anatany of Criticism. Princeton University Press, Princeton.

GARCIAA-BEDOYA M., Carlos (1999) : «La Celestina en la encrucijada». Alma Mater 17: pp. 5-17.

GIIMAN, Stephen (1993) : La novela según Cervantes. Fondo de Cultura Económica, México.

LUKÁCS, Georg (1971): The Theory of the Novel. The Massachussets Institute of Technology Press, Cambridge.

MARAVALL, José Antonio (1975) : La cultura del Barroco. Ariel, Barcelona. MARTIINEZ BONATI, Félix (1992) Don Quixote and the Poetics of the Novel. Comell University Press, Ithaca.

OROZCO DÍAZ, Emilio (1992) : Cervantes y la novela del barroco. Universidad de Granada, Granada.

REIS, Carlos y Ana Cristina M. Lopes (1996) : Diccionario de narratología. Ediciones Colegio de España, Salamanca.

RHEY, Edward C. (1990) : Introducción al Quijote. Crítica, Barcelona.

SEGRE, Cesare (1974) : «Costruzioni rettilinee e costruzioni a spirali nel Don Chisciottes. Fn: Ie stutture e il tempo. Einaudi, Turín; pp. 183-219. SPITZER, Leo (1974) : «Perspectivismo lingüístico en el Quijote». En: Lingüística e historia literaria. Gredos, Mbdrid; pp.135-187. WATT, Ian (1999): Mitos del individualismo modemo. Fausto, Don Quijote, Don Juan y Robinson Crusoe. Cambridge University Press, Madrid. 